einer Ostrea nie traut, weil an beiden Orten sebon wiederholt schlimme Zufälle durch diese Thiere veranlasst worden sind. Krankheit der Thiere überhaupt und nach ihrer Laichzeit insbesondere, Aufenthalt in Kloaken, die für den Abzug von Unrath in's Meer bestimmt gewesen, Ansatz an gekupferten Schiffsböden hat man als die wahrscheinlichen Bedingungen dieser Ausnabmsfälle bezeichnet.

\title{
XV.
}

\section{Die Ausdehnung des Gebietes der giftigen Miess- muscheln und der sonstigen giftigen Seethiere in Wilhelmshaven ').}

(Aus dem pathologisehen Institut zu Berlin.)

Von Professor Dr. Max Wolff in Berlin.

Die nachfolgende Mittheilung schliesst sich an die im October vorigen Jahres in Wilhelmshaven beobachtete, so grosses und berechtigtes Aufsehen erregende Massenvergiftung durch Miessmuscheln an. Ich legte mir nehmlich, nachdem die Giftigkeit der Miessmuscheln im Ganzen und insbesondere der Leber durch vielfache Experimente sicher gestellt worden war, die weitere Frage vor, ob das Giftigwerden der Miessmuscheln nur als ein alleinstehendes Factum anzusehen ist, oder ob ausser den Muscheln auch noch andere Wasserbewohner in Wilhelmshaven giftige Eigenschaften besitzen, und ob sich eventuell Beziehungen zwischen letzteren und den giftigen Muscheln nachweisen liessen. Zur Jüsung dieser vom praktischen und theoretischen Standpunkte gleich wichtigen Frage habe ich Objecte von sehr verschiedenen Punkten der Wilhelmshavener Anlage untersucht, und da sich hierbei, abhängig von dem Fundorte, sehr wesentliche Differenzen in der Wirkung der Untersuchungsobjecte herausstellten, möchte ich zur Orientirung nur einige Punkte über die Wilhelmshavener Anlage voranschicken.

Aus der Jade kommt man zunächst in die etwa $90 \mathrm{~m}$ breite Hafeneinfahrt, von der man in den $120 \mathrm{~m}$ breiten Vorhafen gelangt; 1) Nach einem Vortrage, gehalten in der Berliner medicinischen Gesellschaft. 
A Hafeneinfabrt.

a erste Schleuse.

B Vorhafen.

b zweite Schleuse.

C Ausrüstungs-Bassin.

D Hafen-Kanal.

E Hafen für Bagger und Prähme.

c grosse Schiffbrücke.

d kleine Schiffbrücke.

1: Hafen-Bassin.

G Boots- u. Masten-Hafen.

e Dock I.

$\mathrm{f}$ - II.

g - III.

h Helling $\mathrm{I}$.

i - II.

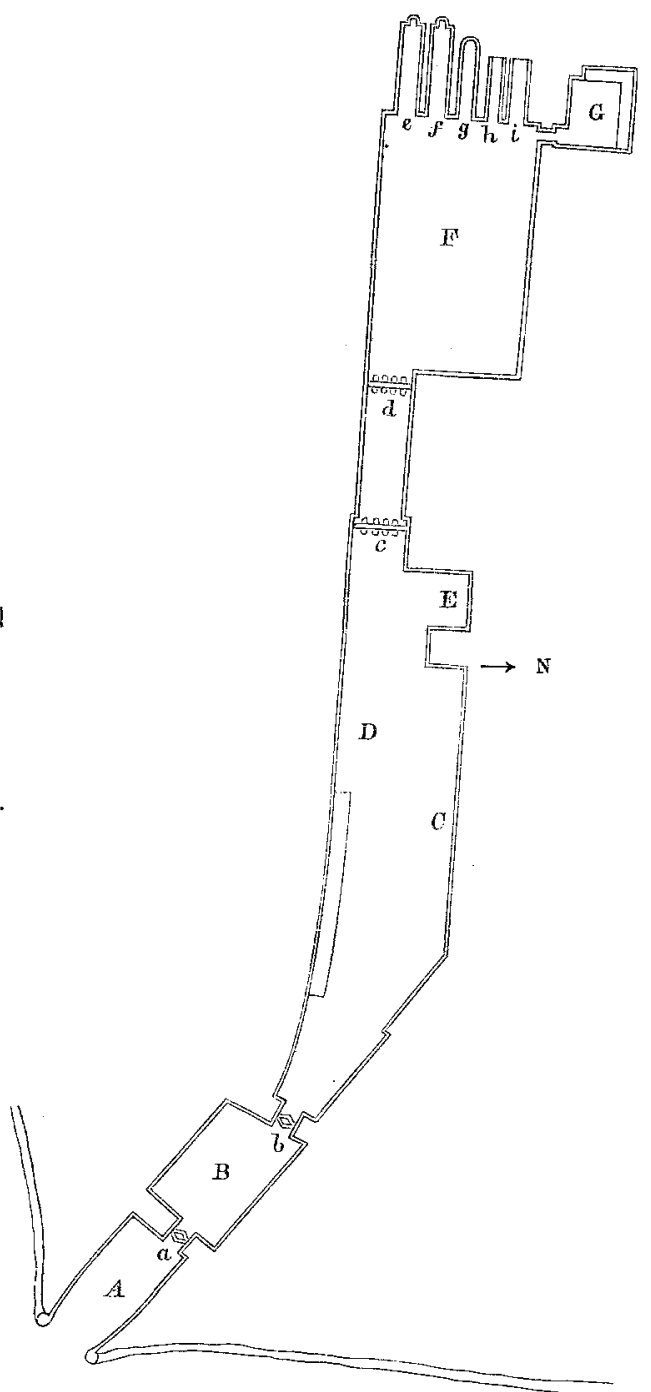

Die Jade

dann folgt der Hafenkanal, etwa $80 \mathrm{~m}$ breit, der schliesslich durch 2 Schiffbrücken, die grosse und die kleine, in das $370 \mathrm{~m}$ lange und $230 \mathrm{~m}$ breite Hafenbassin mit den Docks und Hellingen führt. Der Hafenkanal hat an seiner nördlichen Seite verschiedene Erweiterungen; ebenso hat das grosse Hafenbassin eine 
nordwestliche Erweiterung, den Boots- und Mastenhafen. Es ist nun von besonderer Wichtigkeit hervorzuheben, dass zwischen der Hafeneinfahrt und dem Vorhafen einerseits und zwischen letzterem und dem Hafenkanal andererseits Schleusen angelegt sind, durch welche das ganze Binnenwasser, also vom Vorhafen bis zum Hafenbassin stauend, auf gewöhnlicher Fluthhöhe gehalten wird. Die Schleusen werden nur geöffnet, wenn Schiffe ein- oder ausfahren. In Folge dessen wird, wenn keine rege Schifffahrt vorhanden ist, oder wenn kein Wasser aus anderen Gründen, z. B. zur Spülung der städtischen Abflusskanäle, aus dem Binnenhafen entnommen wird, das Wasser im Binnenhafen im Wesentlichen als ein stagnirendes anzusehen sein. Diese Stagnation ist im Hafenbassin am grössten, vielleicht eine vollkommene; dieselbe nimmt ab, je mehr man sich dem Vorhafen nähert; hier wird der Wasserwechsel beim Oeffnen und Schliessen der Schleusen immerhin ein etwas grösserer sein. Dass das Binnenwasser nicht an den Fluth- und Ebbeströmungen des Jadebusens Theil nimmt, ist nach dem Gesagten selbstverständlich. In Rücksicht auf die später zu erörternden ätiologischen Momente muss ich hier gleich noch hinzufügen, dass die Stadt Wilhelmshaven kanalisirt ist, und dass aus der Stadt oder Umgebung keinerlei Abflüsse in den Binnenhafen münden, sondern dass alle Abflüsse der Stadt direct in die Jade geleitet werden. In gleicher Weise ist eine Verunreinigung des Binnenhafens durch Abfälle aus den Schiffen oder durch Faecalien durch strenge Vorschriften verboten; es existiren reichliche Cloakenanlagen am Strande für die Mannschaften der Schiffe und die Arbeiter.

Ich habe nun, wie bereits oben erwähnt, von sehr verschiedenen Stellen des Wilhelmshavener Binnenwassers verschiedenartiges Material bekommen und geprüft.

I. Die erste Sendung vom 6. Januar dieses Jahres enthielt Miessmuscheln, verschiedene Species von Fischen, ferner eine Krebsart und schliesslich Seesterne.

Die von Herrn Professor F. Eilhard Schulze vorgenommene zoologische Bestimmung hat ergeben, dass es sich bei den übersandten Fischen um 4 verschiedene Arten handelte, um Gadus merlangus Wittling, Osmerus eperlanus Stint, Gobius minutus Weissgrundel, Pleuronectes platessa Scholle. Unter den Seekrebsen 
war nur eine Art vertreten, Crangon vulgaris, die Garneele, die an den Küsten von Deutschland, Holland und Frankreich vielfach gegessen wird; unter den Seesternen war ebenfalls nur eine Art vertreten, Asterias rubens. Der Fundort ist von Wichtigkeit. Nur die Muscheln dieser Sendung stammen von der kleinen Schiffbrücke, also dicht beim Hafenbassin her; die Fische, Garneelen und Seesterne sind theils in der Nähe der grossen Schiffbrïcke, theils am östlichen Ende des Hafenkanals vor der zweiten Schleuse gefangen worden. Muscheln und Seesterne kamen lebendig hier an, die Fische und Garneelen waren todt, aber doch vollkommen frisch.

Das Material wurde nun in ganz übereinstimmender Weise verarbeitet, indem von jeder Species je ein alkoholischer Auszug in der Art, wie es Salkowski gethan (,zur Kenntniss des Giftes der Miesmuschel" d. Archiv Bd. CII. S. 578), und ein wässriger Auszug gemacht und geprüft wurde. Es wurden zu dem Zweck 150-200 g zunächst von jeder Fischart und von den Garneelen möglichst fein zerhackt. Der erhaltene Brei wurde alsdann mit gleichen Quantitäten Alkohol, bez. Wasser übergossen, einen oder einige Tage unter wiederholtem Umschütteln im Kolben stehen gelassen und dann 1-11 Stunden auf dem Wasserbade im Kolben digerirt. Der Alkohol, bez. das Wasser wurden sodann abfiltrirt. Die Filtrate wurden schliesslich auf Schalen über dem Wasserbade bis zur dickflüssigen Consistenz eingedampft, und der Rückstand jedesmal mit $10 \mathrm{ccm}$ sterilisirten Wassers verrieben.

Ueber das Resultat der Fisch-Injectionen kann ich mich kurz fassen. Weder das alkoholische noch das wässrige Extract der Fische brachte jemals Intoxicationserscheinungen hervor, wiewohl die injicirte Quantität im Verhältniss zur toxischen Dosis der Miessmuscheln eine ausserordentlich viel grössere war. Mittelgrosse Meerschweinchen zeigten niemals Vergiftungserscheinungen, auch nicht, nachdem 3-4 Pravaz'sche Spritzen der Auszüge, entsprechend ungefähr $60-80 \mathrm{~g}$ Fischsubstanz, bei jedem. Thiere zur Injection gelangt waren.

Nur bei dem alkoholischen Auszug der Garneelen - der wässrige Auszug war auch hier unwirksam - habe ich einmal bei einem Meerschweinchen Intoxicationserscheinungen gesehen, 
die zwar schnell vorübergingen, aber doch an Miessmuschel-Intoxication erinnerten. Das Thier duckte sích, nachdem es vorher unruhig gewesen und leichte Zuckungen gezeigt, $\frac{1}{2}$ Stunde nach der Injection zu Boden, senkte dabei den Kopf, wie man es in sehr charakteristischer Weise bei den Miessmuschel-Vergiftungen zu sehen bekommt, und zeigte darauf Lähmung der Hinterbeine. Alle diese Erscheinungen gingen aber in kurzer Zeit vorüber, nnd auch erneute Injectionen brachten kein Bild schwererer Vergiftung hervor.

Ganz anders, als bei den eben besprochenen Versuchen, stellten sich die Verhältnisse nach Injectionen mit den Auszügen der Seesterne, auf die ich jetzt näher eingehen muss.

A. Alkoholischer Auszug der Seesterne.

3 grössere Seesterne im Gewicht von $70 \mathrm{~g}$ werden zepquetscht und von dem erhaltenen Brei ganz in der oben beschriebenen Weise ein alkoholischer Auszug gemacht. Der Auszug wird alsdann auf dem Wasserbade eingedampft, wobei sich ein gelbbrauner, reichlich mit schwarzbraunen Oeltropfen vermischter Rückstand ausscheidet, der an ähnliche Ausscheidungen, auch dem Geruche nach, bei ebenso behandelten Miessmuscheln erinnert. Der Rückstand wird mit $10 \mathrm{ccm}$ Wasser verrieben und auf seine Wirksamkeit an Meerschweinchen geprüft.

Versuch 1. Ein Meerschweinchen von $420 \mathrm{~g}$ Gewicht bekommt hente von diesem Auszug 4 Pravaz'sche Spritzen voll injicirt. In den ersten Minuten ist dem Thier nichts Besouderes anzumerken, nach 6 Minuten wird es unrubig, bekommt leichte Dyspnoe, nach 10 Minuten sinkt der Kopf zu Boden, bald darauf duckt sich das ganze Thier nieder, dann werden die vorderen und hinteren Extremitäten gelühnt, die Dyspvoe wirủ sehr stark and nach 20 Minuten erfolgte der Tod unter paralytischen Erscheinungen. Section: In den inneren Organen keine Veränderung.

Versuch 2. Einem Meerschweinchen, $255 \mathrm{~g}$ schwer, werden zunächst $1 \frac{1}{2}$ Pravaz'sche Spritzen voll desselben alkobolischen Auszuges subcutan beigebracht. Da nach Ablauf einer halben Stunde keine deutliche Wirkung eingetreten ist, bekommt dasselbe Thier noch 1 Pravaz'sche Spritze voll injicirt. 5 Minuten später treten Unruhe und leichte Zuckungen auf. 55 Minuten nach Beginn des Versuchs erfolgt Läbmung der hinteren und vorderen Extremitäten, so dass das Tthier kei Bewegungsversuchen ausgleitet. Tod unter' 
Dyspnoe nach $1 \frac{1}{2}$ Stuaden. Section: Keine Veränderung in den inneren Organen.

Versuch 3. Meerschweinchen, $705 \mathrm{~g}$ schwer, bekomwt 4 Pravaz'sche Spritzen yoll subcutan beigebracht. Der Tod erfolgte nach $2 \frac{1}{2}$ Stunden unter paralytischen Erscheinungen und starker Dyspnoe.

Versuch 4 und 5 . $Z$ u diesen Versuchen werden 3 andere Seesterne derselben Sendung und von. fast genau demselben Gewicht ganz ebenso verarbeitet, wie bei den bisherigen Versuchen. 2 Meerschweinchen im Gewicht von $520 \mathrm{~g}$ und $\$ 40 \mathrm{~g}$ bekommen davon je 4 Pravaz'sche Spritzen voll injicirt. Nach 3 Minuten sitzen die Thiere ruhig da, nach 7 Minuten sind die Hinterextremitäten so gelähmt, dass die Thiere dieselben auch beim stärksten Druck nicht fortzuziehen vermögen; bald darauf sind auch die Vorderpfoten gelähmt. Nach 15 Minuten können die Thiere nur mit grösster Mühe sich wieder aufrichten, wenn man sie auf die Seite oder den Räcken legt. Bereits vorher ist der Kopf herabgesunken und Dyspnoe eingetreten. In diesem Zustande liegen beide Thiere ruhig ohne Schmerzensäusserung da, und es erfolgt der Tod bei beiden Thieren unter vollkommener Lähmung fast gleichzeitig $\frac{5}{4}$ Stunden nach der Injection. Section: Bei beiden Thieren ist auffallend eine starke venöse Hyperämie des Peritonaeum parietale und viscerale.

\section{B. Wässriger Auszug der Seesterne.}

Ganz ebenso, wie für den alkoholischen Auszug, werden hier 3 grössere Seesterne im Gewicht von $70 \mathrm{~g}$ zu dem wässrigen Auszug verarbeitet. Der wässrige Auszug wird schliesslich über dem Wasserbad eingedampft auf etwa $10 \mathrm{ccm}$ und ohne weiteren Wasserzusatz zur Injection verwandt.

Versuch 6. Ein Meerschweinchen von $270 \mathrm{~g}$ Gewicht bekommt 3 Pravaz'scbe Spritzen voll von diesem Auszug injicirt. Erfolg: Weder an demselben, noch am folgenden Tage irgend welche Intoxicationserscheinungen.

Versuch 7. Ein Meerschweinchen von $510 \mathrm{~g}$ bekommt 6 Pravaz'sche Spritzen des wässrigen Extracts subcutan injicirt. Das Thier ist nach 5 Minuten etwas unruhig, bekommt nach 10 Ninuten leichtes Zittern, nach 30 Minuten sind die Hinterpfoten leicht paralytisch. Nach 1 Stunde ist die Parese der Hinterextremitäten vorüber, und das Thier 2 Stunden nach der Injection ganz frei von Krankbeitserscheinungen.

Im Gegensatz zu den Injectionen mit den Fischextracten zeigen also die vorstehenden Versuche, dass die Seesterne $z$ weifellos giftig wirken und Intoxicationserscheinungen hervorbringen, die vollkommen denen gleichen, die wir nach Vergiftungen mit Miessmuscheln gesehen haben. 
Die zur Erzeugung dieser Vergiftungen gebrauchte Dosis des alkoholischen Auszuges (Versuch 1-5) schwankte zwischen $2 \frac{1}{2}$ und 4 Pravaz'schen Spritzen; berechne ich diese Dosis des alkoholischen Auszuges auf ganze Seesterne, so brachten hiervon $17 \frac{1}{2}-28 \mathrm{~g}$ die charakteristischen Intoxications-Erscheinungen und den Tod der 'Thiere hervor; berechne ich diese Injectionsdosis nur auf die. Weichtheile der Seesterne, nach Abzug des Skelets, so genügten $10-17 \mathrm{~g}$, um die erörterten Erscheinungen von Paralyse und Dyspnoe auszulösen. Viel weniger giftig wirkte der wässrige Auszug der im Uebrigen ebenso behandelten und in gleicher Quantität verarbeiteten Seesterne (Versuch 6 und 7). Hiervon brachten in einem Fall (Versuch 6) 3 Pravaz'sche Spritzen voll, entsprechend $21 \mathrm{~g}$ ganzer Seesterne, bez. $13 \mathrm{~g}$ Weichtheile, gar keine Intoxicationserscheinungen hervor; in einem zweiten Fall (Versuch 7) lösten 6 Pravaz'sche Spritzen, entsprechend $42 \mathrm{~g}$ ganzer Seesterne, bez. $26 \mathrm{~g} \mathrm{Weich} \mathrm{theile,} \mathrm{nur} \mathrm{leicht} \mathrm{paretische,} \mathrm{schnell} \mathrm{vorüber-}$ gehende Erscheinungen aus. Dieser bemerkenswerthe Unterschied zwischen den wässrigen und den alkoholischen Extracten in der Giftwirkung war die Veranlassung, dass ich in der Folge nur noch mit den alkoholischen Extracten der Seesterne arbeitete.

Das Material dieser Sendung I stammte von verschiedenen Fundorten her. Ich hatte dasselbe gemischt bekommen und so geprüft. Ein Theil der Seesterne war, wie oben bemerkt, in der Nähe der grossen Schiffbrücke, also am westlichen Ende des Hafenkanals, der andere Theil über $800 \mathrm{~m}$ davon entfernt vor der zweiten Schleuse, also am östlichen Ende des Hafenkanals gefangen worden. Die weiteren Versuche sub II und III lassen keinen Zweifel darüber, dass der giftige Antheil an diesem gemischten Material den Seesternen, die von der grossen Schiffbrücke herstammten, zukommt.

II. Am 20. Januar dieses Jahres erhielt ich eine 2. Sendung. von Fischen, Seesternen und Miessmuscheln, die nur am östlichen Ende des Hafenkanals, vor der zweiten Schleuse, gefangen waren. Die Fische, unter denen wiederum Stint, Weissgrundel, Scholle neben einzelnen Garueelen vertreten waren, erwiesen sich bei vereinzelten, damit angestellten Versuchen, wie 
früher, wirkungslos. Eine grössere Versuchsreihe wurde mit den Seesternen ausgeführt.

$450 \mathrm{~g}$ Seesterne, deren Weichtheile nach Entfernung des Skelets ungefähr $240 \mathrm{~g}$ betrugen, waren Tags zuvor zu einem Brei zerquetscht und mit $800 \mathrm{ccm}$ Alkohol übergossen. Nach 24 Stunden wurde der alkoholische Auszug in der mehrfach erörterten Weise weiter verarbeitet und schliesslich eingedampft, wobể sich eine zähflüssige schwarzbraune Masse, dieses Mal mit nur spärlichen Oeltropfen vermischt, ausscheidet. Dieser zähflüssige Rückstand wird durch Zusatz von $100 \mathrm{ccm}$ Wasser auf etwa $130 \mathrm{~cm}$ gebracht und zu nachfolgenden Injectionen verwandt.

22. Januar 1886, Versuch 9 und 10. Zwei Meerschweinchen, $570 \mathrm{~g}$, bez. $545 \mathrm{~g}$ schwer, bekommen zunächst 4 , und da nach $\frac{1}{2}$ Stunde beinerlei Erscheinungen eingetreten sind, noch 2 Pravaz'sche Spritzen subcutan injicirt. Diese 6 Pravaz'sehe Spritzen alloholischen Auszuges, die ca. je $20 \mathrm{~g}$ ganzer Seesterne oder je $11 \mathrm{~g}$ Weichtheilen letzterer entsprechen, bleiben ohne jede toxische Wirkung innerbalb 24siündiger Beobachtungszeit.

Versucb 11 und 12. Von demselben Alkoholauszug erhalten? Kaninchen je $50 \mathrm{cem}$, entsprechend je $173 \mathrm{~g}$ ganzer Seesterne oder je $92 \mathrm{~g}$ Weichtheilen, in den Magen injicirt. Beide Kaninchen sind sowoh! an demselben, wie am folgenden Tage völlig munter.

Versuch 13-15. Von $392 \mathrm{~g}$ Seesternen (34 Stück), deren Weichtheile nach Abzug des Skelets annähernd $192 \mathrm{~g}$ wiegen, wird ein neuer alkobolischer Auszug hergestellt, der bis zur Syrupsconsistenz eingedampft und mit Wasser verrieben wird. Hiervon bekommen 3 Meerschweinchen ınit einem Körpergewicht von $637 \mathrm{~g}, 605 \mathrm{~g}$ und $377 \mathrm{~g}$ im Laufe einer Stunde je 6, 5, 4 Pravaz'sehe Spritzen voll injieirt. Nach dieser grossen Dosen, die ungefähr $39,32,26 \mathrm{~g}$ ganzer Seesterne, bez. $19,16,13 \mathrm{~g}$ Wejchtheilen letzterer entsprechen, sind keinerlei Vergiftungserscheinungen zu beobachten.

Im Gegensatz zur Sendung I haben sich nach den vorstehenden Versuchen die Seesterne der Sendung II völlig wirkungslos erwiesen, wiewohl die beigebrachte Dosis mehrfach erheblich grösser gewesen ist, als bei Sendung I. Die subeutan heigebrachte Dosis schwankte bei den 5 Versuchsthieren zwischen 4 und 6 Pravaz'schen Spritzen, entsprechend 20-39g ganzer Seesterne, bez. 11-19g Weichtheilen derselben. Auch vom Magen aus wirkten diese Seesterne nicht: bei 2 Kaninchen sind jedesmal ca. $173 \mathrm{~g}$ ganzer Seesterne, bez. $92 \mathrm{~g}$ Weichtheile ohne toxische Wirkung geblieben. 


\section{8}

Wir müssen hiernach die Seesterne der Sendung II, deren Fundort das östliche Ende des Hafenkanals vor der zweiten Schleuse war, für ungiftig erklären.

III. Ich schliesse hier gleich noch das Resultat der Versuche 16-21 mit einer III. Sendung an, die ich später, am 12. Februar 1886, von demselben Fundorte, also dem östlichen Ende des Hafenkanals, erhalten habe.

$400 \mathrm{~g}$ Seesterne dieser Sendung (= 32 Stück) mit einem Gewicht der Weichtheile von etwa $190 \mathrm{~g}$ wurden zu dieser Versuchsreihe verwandt. Von dem alkoholischen Auszuge bekamen zunächst 3 Meerschweinchen subcutan beigebracht 4-6 Pravaz'sche Spritzen, entsprechend $26 \frac{2}{3}, 33 \frac{1}{3}$, $40 \mathrm{~g}$ ganzer Seesterne, bez. $12 \frac{2}{3}, 15 \frac{5}{6}, 19 \mathrm{~g}$ der Weichtheile. Kein einziges Thier zeigte hiernach Intoxicationserscheinungen.

Sodann wurden 3 Kaninchen ebenfalls ganz ohne Erfolg injicirt mit

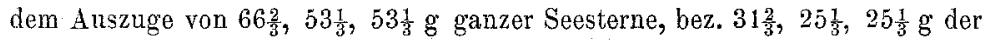
Weichtbeile.

Auch nach den vorstehenden Resultaten (Versuch 16-21) müssen also, in Uebereinstimmung mit den Ergebnissen sub II, die Seesterne vom östlichen Ende des Hafenkanals für ungiftig gelten, da selbst die noch erheblich grösseren Injectionsdosen dieser Versuchsreihe keine Intoxicationserscheinungen hervorbrachten.

IV. Aus den weiter östlichen Wassergebieten von Wilhelmshaven waren hiernach neue Aufschlüsse über die Seesterne, deren Giftigkeit sub I nachgewiesen war, nicht zu erwarten; ich prüfte daher von jetzt ab die Seesterne aus dem westlichen Theile des Wilhelmshavener Gebietes, aus dem Hafenbassin, bez. den Anhängen desselben.

Am 31. Januar erhielt ich eine 4. Sendung von Seesternen und Muscheln aus dem „Bootshafen", einer nordwestlichen Erweiterung des Hafenbassins; Muscheln und Seesterne noch lebend.

Am 1. Februar wurden. $350 \mathrm{~g}$ dieser Seesterne, deren Weichtheilgewicht annähernd $200 \mathrm{~g}$ beträgt, in der gewöhnlichen Weise verarbeitet und mit dem daraus hergestellten alkoholischen Extract die nachfolgenden Injectionsversuche gemacht:

Versuch 22. Am 2. Februar bekommt ein Meerschweinchen von $600 \mathrm{~g}$ Körpergewicht 4 Pravaz'sche Spritzen voll des alkoholischen Extractes injicirt, entsprechend $23 \frac{1}{3} \mathrm{~g}$ ganzer Seesterne, bez. $13 \frac{1}{3} \mathrm{~g}$ Weichtheilen. Das Thier zeigt hiernach nach 8 Minuten Unruhe, leichte Zuckungen, Athem- 
noth, nach 10 Minuten sinkt der Kopf herab, hald darauf duekt sich das ganze Thier zu Boden; nach 15 Minuten Lähmung der Vorderpfoten und Hinterpfoten, so dass das Thier bei Bewegungsversuchen ansgleitet; zunehmende Dyspnoe; der Tod erfolgt 30 Minuten nach der Injection bei totaler Paralyse.

Versuch 23 und 24. Zwei Meerschweinchen im Gewicht von 560 und $390 \mathrm{~g}$ werden ebenfalls je 4. Praraz'sche Spritzen obigen Auszuges subcutan injicirt. No. 23 war nach $\frac{3}{4}$ Stunden, No. 24 schon nach 15 Minuten todt unter ganz denselben paralytischen Erscheinungen, wie No. 22.

Versueh 25. Ein Meerschweinchen von $300 \mathrm{~g}$ Körpergewicht erhält nur 2 Pravaz'sche Spritzen des Auszuges, entsprechend $11 \frac{2}{3} \mathrm{~g}$ ganzer Seesterne, bez. $6 \frac{2}{3} g$ Weichtheilen. Nach 6 Minuten Unrube, leichte Zuckungen; nach 10 Minuten Sinken des Kopfes, Lähmung der Extremitäten, Dyspnoe. Tod unter paralytischen Erscheinungen nach 20 Minuten.

Am 3. Februar wird ein neues Extract der Seesterne von Sendung 4 hergestellt. Es werden dieses Mal nur 5 Seesterne mit einem Gewicht von nur $108 \mathrm{~g}$ incl. Skelet und annähernd nur $74 \mathrm{~g}$ Weichtheilen nach Abzug des Skeletrückstandes zur Extraction verwandt und ausserdem viel kleinere Dosen, als bei den vorigen Versuchen, injicirt.

Versuch 26 und 27. Zwei Meerschweinchen mit einem Körpergewicht

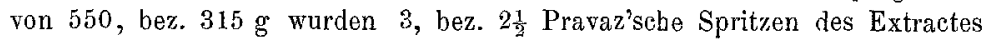
subcutan injicirt. Diese Dosen entsprechen $10 \frac{4}{5} \mathrm{~g}$ und $9 \mathrm{~g}$ ganzer Seesterne, bez. $7 \frac{2}{5}$ und $6 \frac{1}{6} \mathrm{~g}$ Weichtheilen derselben. Meerschweinchen No. 26 bekommt nach 8 Minuten Dyspnoe, duckt sich zu Boden, ]ässt den Kope fallen und ist nach 20 Minuten total gelähmt. Darauf erfolgen noch schwache Zuckungen und unter stärkster Dyspnoe nach 25 Minuten der Tod des Thieres.

Meerschweinchen No. 27 ist unter gleichen Erscheinungen bereits nach 10 Minuten todt.

Versuch 28 and 29. Zwei Meersehweinchen bekommen je 2 Pravaz'sche Spritzen voll subcutan entsprechend je $7 \frac{1}{5} \mathrm{~g}$ ganzer Seesterne, bez. $5 \mathrm{~g}$ Weichtheilen. - No. 28 ist nach voraufgehender Unruhe und leichten Zuckungen nach 15 Minuten total gelähmt, und der Tod tritt unter stärkster Dyspnoe nach 20 Minuten ein. Die Section ergiebt Lungenbyperämie und starke Hyperämie des Peritonaeum parietale und viscerale. -

No. 29 ist unter denselben voraufgehenden Erscheinungen bereits nach 5 Minuten gelähmt und nach 8 Minuten todt.

Versuch 30 und 31 . In diesen Versuchen sind noch kleinere Dosen zur Injection gebracht. Meerschweinchen No. 30 mit einem Körpergewicht von $220 \mathrm{~g}$ erhält $6 \frac{3}{10} \mathrm{~g}$ ganzer Seesterne, bez. $4 \frac{1}{3} \mathrm{~g}$ Weichtheile: Unruhe, Dyspnoe, Lähmung aach 10 Minuten, Tod unter Paralyse nach 20 Minuten. 
Heerschweinchen No. 31 von einem Körpergewicht von $630 \mathrm{~g}$ wird sogar schon nach Beibringung von $5 \frac{2}{5} \mathrm{~g}$ gauzer Seesterne, bez. $3 \frac{7}{10} \mathrm{~g}$ Weichtheilen nach 5 Minuten unrubig, dyspnoetisch, rach 10 Minuten gelähnt und stirbt unter stärkster Dyspuoe nach 25 Minuten. Section: Starke Lungenbyperämie und starke Hyperämie der Peritonäalgefässe.

In gleicher Weise, wie Meerschweinchen, gehen auch Kaninchen zu Grunde.

Kaninchen No. 32 und 33 mit einen Körpergewicht von 1470, bez. $1200 \mathrm{~g}$ bekommen im Laufe einer Stude in $Z$ wischen räursen je $4 \frac{1}{2}$, be\%. 4 Pravaz'sche Spritzen des Auszuges, d. h. entsprechend je 14 und $16 \mathrm{~g}$ ganzer Seesterne, bez. 11 und $9 \mathrm{~g}$ der Weicltheile derselben beigebracht. 70 Minuten nach der letzten Injection liegen beide Thiere am Boden mit gesenktem Kopf; dann tritt Lähmung ein, die Thiere können sich nicht mehr aufrichten, Tod bei beiden Thieren 2 Stunden nach der ersten Injection unter stärkster Dyspnoe.

Kaninchen No. 34 von $1100 \mathrm{~g}$ Körpergewicht bekomwt auf einmal 2 Pravaz'sche Spritzen voll injicirt, entsprechend $7 \frac{1}{5} \mathrm{~g}$ ganzer Seesterne, bez. $5 \mathrm{~g}$ Weichtheilen. Nach 20 Minuten Unruhe, starke Dyspnoe, nach 25 Minuten Herabsinken des Kopfes, Niederducken des ganzen Thieres. Nach 35 Minuten füllt das Thier zur Seite, Tod nach 40 Minuten. Section: Beide Lungen stark hyperämisch.

Die vorstehenden Versuche sub IV mit den Seesternen aus dem Bootshafen schliessen sich also hinsichtlich der Wirkung der Seesterne an die Sendung No.I an und stehen im Gegensatz zu den Seesternen der Sendung II und III. Die Seesterne aus dem Bootshafen haben sich übereinstimmend in 13 Versuchen bei Kaninchen und Meerschweinchen als sehr giftig, durchweg letal wirkend erwiesen. Die kleinsten noch letal wirkenden Dosen des alkoholischen Auszuges, die wir angewandt haben, waren beim Meerschweinchen entsprechend $5 \frac{2}{5}$ und $6_{\frac{3}{10}} \mathrm{~g}$ ganzer Seesterne, bez. $\frac{3}{10}$ und $4 \frac{1}{3} \mathrm{~g}$ Weichtheilen der Seesterne. Bei den an Körpergewicht viel schwereren Kaninchen war die kleinste, von uns angewandte, letal wirkende Dosis des alkoholischen Auszuges entsprechend $7 \frac{1}{5} \mathrm{~g}$ ganzer Seesterne, bez. $5 \mathrm{~g}$ Weichtheilen derselben.

Die Symptome der Seesternvergiftung waren in allen Fällen denen der Muschelvergiftung überraschend ähnlich, wie die obigen Versuche beweisen.

V. Am 17. Februar 1886 bekam ich eine 5. Sendung Seesterne, die von einer anderen stelle herstammten, von dem 
"Eingang des Hafenbassins in den Bootshafen". Der ganze Fang bestand nur aus 6 kleineren Seesternen, die vach vieler Mühe gewonnen wurden, da die Oberfläche des Wassers mit Eis bedeckt war. Von 5 Seesternen mit einem Gewicht von $47 \mathrm{~g}$, bez. $27 \mathrm{~g}$ Weichtheilen, nach Abzug des Skelets, wird in der gewöhnlichen Weise der alkoholische Auszug gemacht.

18. Februar. Versuch 35. Ein Meersehweinchen von $330 \mathrm{~g}$ Körpergewicht bekommt unter die Haut $2 \frac{1}{2}$ Pravaz'sche Spritzen voll des Auszuges entsprechend $7 \frac{5}{6} \mathrm{~g}$ ganzer Seesterne, bez. $4 \frac{1}{2} \mathrm{~g}$ Weichtheilen derselben: Nach vorangehender Unruhe und leichten Zuckungen sinkt nach 15 Minuten zunächst der Kopf herab, bald darauf duckt das ganze Thier sich nieder und ist. nach weiteren 5 Minuten total gelähmt. Unmittelbar vor dem Tode wieder schwache Zuckungen, während die Dyspnoe stark zunimmt. 37 Minuten nach der Injection Tor.

Versuch 36. Meerschweinchen, $275 \mathrm{~g}$ schwer; Injectionsdosis 2 Pravaz'sche Spritzen $=6 \frac{1}{4} \mathrm{~g}$ ganzer Seesterne, bez. $3 \frac{3}{5} \mathrm{~g}$ Weichtheilen. Das Thier sitzt bereits 5 Ninuten nach der Injection zusammengekauert mit herabgesunkenem und seitwärts geneigtem Kopfe da; 5 Minuten später Zuckungen, nach weiteren 10 Minuten ist das Thier total gelähmt, liegt mit stärkster Dyspnoe auf der Seite, kann sich nicht aufrichten. Tod tritt 27 Minuten nach der Injection ein.

Versueh 37 . Kanincben, $1027 \mathrm{~g}$ schwer. Injectionsdosis 2 Pravaz'sche Spritzen $=6 \frac{1}{4} \mathrm{~g}$ ganzer Seesterne, bez. $3 \frac{3}{5} \mathrm{~g}$ Weichtheilen. Nach voraufgehender Unruhe, Dyspnoe, leichten Znckungen liegt das Thier 30 Minuten nach der Injection mit gesenktem Kopf, weit ausgestreckten Extremitäten total gelähmt da, so dass es beim Umlegen auf die Seite oder auf den Rücken sich nicht wieder aufrichten kann. Tod etwa $1 \frac{1}{2}$ Stunden nach der Injection unter stärkster Dyspnoe.

Versuch 38. Kaninchen, $1300 \mathrm{~g}$ schwer, bekomint zunächst ebenfalls 2 Pravaz'sche Spritzen voll injicirt. Da nach 40 Minuten nur mässige Frscheinungen von Unruhe eingetreten sind, bekommt das Thier noch 1 Pravaz'sche Spritze voll injicirt, so dass es also im Ganzen $9 \frac{2}{5} \mathrm{~g}$ ganzer Seesterne, bez. $5 \frac{2}{5} \mathrm{~g}$ Weichtheile derselben bekommen hat. 5 Minuten nach der letzten Injection Zuckungen, dann zunehmende Dyspnoe und 30 Minuten nach der letzten Injection totale Lähmung der Extremitäten. In diesem gelähmten Zustande liegt das Thier $4 \frac{1}{2}$ Stunde. Am nächsten Morgen war das Thier zwar noch matt, aber hatte sich doch sonst erholt; Lähmungen nicht nehr vorhanden.

Zwei Tage später wude bei demselben Kaninchen ganz derselbe Symptomencomplex noch einmal und zwar nach derselben Injectionsdosis des alkoholischen Extractes hervorgebracht. Das Thier blieb auch dieses Mal am Leben. 
Die vorstehenden Versuche sub $V$ beweisen also, dass die Seesterne auch von einer anderen Stelle des Hafenbassins, dem "Eingange des Hafenbassins in den Bootshafen", sehr giftig sind. Die in dieser Versuchsreihe durchweg angewandten Dosen sind noch kleiner, als in Versuchsreihe IV. Die kleinste injicirte Dosis des Auszuges entsprach hier $6 \frac{1}{4} g$ ganzer Seesterne, bez. $3 \frac{3}{5} \mathrm{~g}$ Weichtheilen derselben; die grösste Dosis entsprach $9 \frac{2}{5} \mathrm{~g}$ ganzer Seesterne, bez. $5 \frac{2}{5} \mathrm{~g}$ Weichtheilen derselben. Die Vergiftungssymptome waren völlig identisch mit denen, die wir sub IV beobachtet haben.

VI. Fasse ich das Resultat aus allen obigen Versuchen I-V zusammen, so ergeben sich daraus zwei bemerkenswerthe Thatsachen: einmal dass in Wilhelmshaven ausser den Miessmuscheln Wasserbewohner, die einem ganz anderen Thierkreis angehören, mit sehr giftigen Eigenschaften vorkommen, nehmlich Seesterne, und zweitens, dass die giftigen Seesterne nur auf bestimmte Wassergebiete in Wilhelmshaven beschränkt sind.

Was zunächst die Giftigkeit der Seesterne anbetrifft, so sind die durch dieselben erzeugten toxischen Erscheinungen so frappant ähnlich dem Symptomencomplex, den wir von den Miessmuschel-Intoxicationen her kannten, dass die Annahme einer Identitit der toxischen Substanzen in beiden Thierarten keinem berechtigten $Z$ weifel unterliegt. Wie bei den Miessmuscheln, tritt bei den Seesternen nach der Injection von alkoholischem Extract aus 3-5 $\mathrm{g}$ Weichtheilen, bez. $6-8 \mathrm{~g}$ ganzen Seesternen (s. obige Versuche), zunächst Unruhe und Athemnoth der Thiere ein, darauf erfolgt das charakteristische Herabsinken des Kopfes und das Niederducken des ganzen Thieres, dann das Ausgleiten der Extremitäten bei Bewegungsversuchen, bald darauf vollkommene Lähmung der Vorder- und Hinterpfoten and schliesslich bei steigender Dyspnoe Tod unter Erscheinungen allgemeiner Paralyse; leichte convulsivische Erscheinungen kamen selten gegen Ende des Lebens vor. Nur der zeitliche Ablauf der Intoxieationssymptome war bei der Seestern-Vergiftung im Ganzen etwas mehr protrahirt, als wir denselben von den vorjährigen Vergiftungen mit Miessmuscheln her kannten. Auf diesen Punkt kommen wir noch zurück, da auch die jetzigen Miess- 
muscheln sich nach der Richtung hin geändert haben und, ähnlich den Seesternen, ebenfalls keinen so acuten Verlauf der Intoxication mehr zu Stande bringen, wie früher.

In der Literatur habe ich über die angegebene charakteristische Wirkung der Seesterne gar nichts gefunden; dieselbe ist bisher unbekannt geblieben. Ich finde überhaupt nur eine Angabe aus dem Jahre 1862 und zwar in dem Handbueh der Toxikologie von Husemann S. 242, nach der einige Seesterne aus der Ordnung der Asterida (Species Asteria) giftige Eigenschaften besitzen sollen. Chemisch ist nach dieser Angabe nichts Giftiges in ihnen nachgewiesen, "und die ganze Annahme beruht nur auf den Versuchen Breumié's und Durandeau's an Hunden, welche nach Fütterung mit rohen oder gekochten Seesternen heftige, selbst tödtlich endende entzündliche Vergiftungsfillle bekamen". Das ist alles, was ieh über den Gegenstand bei genauer Durchsicht der Literatur gefunden habe.

Was nun die Frage anbetrifft, ob unsere giftigen und ungiftigen Seesterne vielleicht verschiedene Varietäten sind, so liegt die Sache hier ganz ebenso, wie bei den Miessmuscheln. Es fehlen besondere Kenuzeichen der Giftigkeit der einzelnen Exemplare. Nur die Leber der Seesterne zeigte wiederholt, wenn auch ebenfalls nicht constant, einen gewissen Unterschied bei den giftigen im Vergleich mit den nicht giftigen Seesternen, und erinnerte dieses Verhalten ebenfalls an die Muscheln. Ich hatte in meiner Arbeit "die Localisation des Giftes in den Miesmuscheln" (dieses Archiv, Bd.103 S. 187) gegenüber anderweitig angegebenen, meiner Erfahrung nach nicht zutreffenden Merkmalen in Bezug auf Grösse und Consistenz der Leber der Giftinuscheln bemerkt: „Viel häufiger, wenn auch ebenfalls nicht constant, möchte ich den Unterschied in der Färbung hinstellen, jedoch nicht so, dass die Leber giftiger Muscheln dunkler ist, wie Coldstream will, sondern dass sie im Gegentheil heller und zwar heller gelb erscheint als die nicht giftige. Auch mikroskopisch habe ich mehrfach in der Leber solcher giftigen Muscheln reichlicher und in grösserer Menge Pigment angetroffen, als in ungiftigen." Diese Angaben treffen genau auch für das Pigment der Leber der Seesterne zu. 
VII. Der zweite Satz, der sich aus den obigen Vẹsuchen sub I-V ergeben hatte, war der, dass die giftigen Seesterne nur auf bestimmte Wassergebiete in Wilhelmshaven beschränkt sind. Als Wohnplätze der giftigen Seesterne sind nachgewiesen der "Bootshafen", eine nordwestliche Erweiterung des Hafenbassins (s. Versuchsreihe IV), ferner der "Eingang des Hafenbassins in den Bootshafen" (s. Versuchsreihe V) und schliesslich etwa 700 Meter davon entfernt die Nähe der "grossen Schiffbrücke" (s. Versuchsreihe I). An allen diesen Stellen haben sich die Seesterne als charakteristisch giftig und durchweg letal wirkend bei Kaninchen und Meerschweinchen erwiesen. Die angegebenen Wohnplätze der giftigen Seesterne umfassen also die äussersten Enden des Hafenbassins und gehen noch darüber hinaus; es unterliegt daher auch im Hinblick auf die Giftigkeit der Muscheln im Hafenbassin und die vielfachen Analogien zwischen Muscheln und Seesternen der Schluss keinem berechtigten $Z$ weifel, dass die Seesterne auch im Hafenbassin, woher sie nicht $\mathrm{zu}$ erlangen waren, giftige Eigenschaften besitzen.

Allerdings ergab sich bei näherer Betrachtung hinsichtlich der Intensität der toxischen Eigenschaften doch ein gewisser Unterschied der Seesterne von den genannten verschiedenen Wohnplätzen. Die Seesterne aus dem westlichen Theile des Hafenbassins, dem Bootshafen und dem Eingange in letzteren bewirkten selbst bei viel kleinerer Injectionsdosis schneller die charakteristische Vergiftung und den Tod der Thiere, als die Seesterne, die in der Nähe der grossen Schiffbrücke, also mehr östlich gefangen waren.

Diese Abschwächung der Giftwirkung tritt nun in eclatantester Weise hervor, je mehr man sich dem Osten des Wilhelmshavener Wassergebietes nähert. So zeigen die Seesterue vom östlichen Ende deś Hafenkanals vor der zweiten Schleuse, über 800 Meter entfernt von der grossen Schiffbrücke, keinerlei giftige Eigenschaften mehr. Unter 13 Versuchen, die mit diesen Seesternen an Kaninchen und Meerschweinchen angestellt sind, kamen bei keinem einzigen Thiere, weder vom Magen, noch vom subcutanen Gewebe aus, irgend welche Intoxicationserscheinungen zu Stande (s. Versuchsreihe II und III), wiewohl die Injections- 
dosis oftmals um das 8-10fache grösser war, als die von den Seesternen der Anhänge des Hafenbassins.

Die Seesterne am östlichen Ende des Hafenkanals müssen also, nach den wiederholten Versuchen mit verschiedenen Sendungen, für ungiftig gelten, und es ist auch nicht zu erwarten, dass noch weiter östlich gesammelte Seesterne giftige Wirkungen ausüben.

VIII. Es war nun mit Rücksicht auf die vorstehenden Ergebnisse von ganz besonderem Interesse zu untersuchen, ob die Wohnplätze der giftigen Seesterne auch gleichzeitig die Wohnsitze der giftigen Muscheln sind, oder ob sich hier gewisse Differenzen ergeben. Ich habe nun gleichzeitig mit den Seesternen von denselben Fundorten Miessmuscheln bekommen und letztere nach denselben Methoden geprüft, wie die Seesterne.

Hierbei hat sich nach zahlreichen Versuchen ein vollkommen congruentes Verhalten beider Arten von Seethieren herausgestellt. Je weiter nach Osten der Hafenanlage die Miessmuscheln gewonnen waren, um so weniger giftig wirkten dieselben. Wiederholte Prüfungen solcher Muscheln, z. B. von dem östlichen Ende des Hafenkanals vor der zweiten Schleuse, wo auch die ungiftigen Seesterne herstammten, ergaben keine toxische Wirkung, selbst nicht in sehr erheblichen Dosen. Al. kohol-Auszüge dieser Muscheln, subcutan injicirt, in einer Dosis entsprechend 8-10 g Muschelsubstanz, waren bei Kaninchen wirkungslos, wiewohl diese Dosen um das 60--80fache, z. B. die von Salkowski a. a. 0. S. 582 für ein noch kleineres Kaninchen gefundene, letale Dosis vorjähriger Muscheln übertreffen. Hingegen sind die von dem westlichen Theile des Wilhelmshavener Wassergebietes, vom Hafenbassin, bez. seinen Anhängen, der kleinen Schiffbrücke, dem Bootshafen, gefangenen und vielfach geprüften Miessmuscheln durchweg giftig in gleicher Weise, wie die eben daher stammeuden Seesterne.

Die vorstehenden Untersuchungen über die Ausdehnung des Gebietes der giftigen Seesterne oder Miessmuscheln beziehen sich auf die diesjährigen Prüfungen von Anfang Januar bis Mitte Februar. Das giftige Gebiet stimmt aber in der Hauptsache überein auch mit dem vom Herbst vorigen Jahres, insofern auch damals die Muscheln im Hafenbassin und seinen Anh:̈ngen am 
giftigsten waren. Allerdings war Ende vorigen Jahres, als die Giftigkeit der Muscheln überhaupt, ihre grösste Intensität erreicht hatte, das Gebiet derselben ausgedehnter, insofern sich nach Schmidtmann auch noch im Vorhafen giftige Muscheln vorfanden; in letzterem waren dieselben jedoch bereits nur noch schwach giftig, und in der Hafeneinfahrt waren die Muscheln auch damals völlig ungiftig. Es ist also nach unserer Erfahrung in den letzten Monaten eine Beschränkung des Wohnsitzes der giftigen Muscheln eingetreten.

Ich muss nun noch auf einen sehr wesentlichen Punkt eingehen, auf die Abnahme der Giftigkeit der Miessmuscheln uberhaupt, selbst an den giftigsten Stellen in den letzten Monaten. Wenn ich meine zahlreichen Experimente mit Miessmuscheln von Mitte November ungefähr bis Anfangs December vorigen Jahres vergleiche mit den diesjährigen Versuchen vom 6. Januar bis 12. Februar, so kann die Abnahme der Giftigkeit der Miesmuscheln in Wilhelmshaven im Ganzen keinem Zweifel unterliegen. Bei meinen vorjährigen Versuchen (siehe dieses Archiv Bd. CIII. S. 187) war es die Regel, dass Muschel für Muschel giftig wirkte; fast immer stellten sich Unruhe, Athemnoth, Lähmungserscheinungen der inficirten Thiere sofort nach der Beibringung ein, und der ganze Symptomencomplex bis zum Tode der Thiere verlief sehr bäufig in kürzester Frist, innerhalb 2-5 Minuten. Das hat sich sehr geändert. Schon Anfang Januar dieses Jahres fand ich die Muschelwirkung abgeschwächt. Von einer früher sehr giftigen Stelle, der kleinen Schiffbrücke, war jetzt weder Muschel für Muschel mehr giftig, so dass z. B. von der herauspräparirten Leber von 8 Muscheln überhaupt nur 5 und diese langsamer wirksam gewesen sind, noch traten selbst bei erheblich grösseren Dosen der alkoholischen Extracte der ganzen Muscheln die charakteristischen Lähmungserscheinungen und der Tod so schnell, wie bei den vorjührigen Muscheln, ein. Ebenso liegen auch die Verhältnisse bei den Giftmuscheln von einer andern Stelle her, dem "Bootshafen", dessen Muscheln nach einer Mittheilung von Herrn A potheker König in Wilhelmshaven vor 2 Monaten noch sehr giftig gewesen waren. Die Anfangs Februar mit diesen Muscheln vielfach angestellten Versuche haben ebenfalls den Nachweis 


\section{7}

einer Abuahme ihrer Giftigkeit geliefert. Zwar bekommt man mit den jetzigen Muscheln aus dem Bootshafen stets noch den charakteristischen Symptomencomplex der Vergiftung bei Kaninchen und Meerschweinchen, aber auch hier erfolgten, trotzdem niemals kleinere, meist grössere Dosen zur Anwendung kamen, jetzt die Vergiftungserscheinungen viel langsamer, wie früher. Sehr häufig war die Lähmung erst nach 1 Stunde complet, and während die Leber und das Leberextract meiner früheren Muscheln unter allen Umständen schnell tödteten, sind z. B. mit den jetzigen Muscheln des Bootshafens von 13 mit Leberextract injicirten Kaninchen und Meerschweinchen äberhaupt nur 7 gestorben.

Also eine allmählich zunehmende Entgiftung der Muscheln in Wilhelmshaven ist zweifellos. Der Unterschied zwischen jetzt und früher war so überraschend, dass ich mich an Herrn Kreisphysicus Schmidtmann und Herrn Apotheker König in Wilhelmshaven wandte mit der Anfrage, ob sie ähnliche Erfahrungen dort gemacht hätten. Beide Herren hatten die Güte, mir Mitte Februar mitzutheilen, dass sie ebenfalls eine sehr erhebliche Abnahme der Giftigkeit der jetzigen Muscheln constatiren müssten.

Mit dieser allmählichen Entgiftung der Muscheln geht nun aber auch eine andere Thatsache Hand in Hand, oder ist vielmehr als Folge des allmählichen Entgiftungsprozesses der Muscheln zu betrachten. Als wir die Miessmuscheln im November und Anfing December vorigen Jahres, als ihre Giftigkeit am grössten war, untersuchten, konnten wir durch Isolirungsversuche constatiren, dass die Leber allein als Hauptheerd der Giftigkeit der Miessmuscheln anzusprechen ist. Die übrigen Organe brachten damals bei sehr zahlreichen Versuchen nie, weder in Substanz noch in Wasser verrieben, noch in Extractform, und zwar trotz erheblich grösserer Dosen, Vergiftungserscheinungen hervor. Dieses Verhältniss hat sich mit dem jetzt bei den Muscheln vor sich gehenden Entgiftungsprozess einigermaassen geändert. Das in die Leber auf der $\mathrm{H}$ öhe der Giftigkeit der Muscheln abgelagerte oder dort gebildete Gift gelangt bei dem jetzigen Entgiftungsvorgang in die Circulation und kommt hierbei vor der Ausscheidung aus dem Muschelkörper auch in ein oder das andere Organ des letzteren hinein. Es scheinen hier analoge 
Verhältnisse vorzuliegen, wie z. B. bei der Ausscheidung von metallischen Giften (Blei, Quecksilber), die lange Zeit in bestimmten Organen des menschlichen Körpers verweilt haben. Wir haben demzufolge jetzt auch mehrere Mal bei analogen Isolirungsversuchen, wie früher, nicht blos mit den Extracten der Leber, sondern auch mit den Extracten der übrigen Weichtheile charakteristische Intoxicationserscheinungen nachweisen können. Allerdings findet sich auch jetzt noch bei den Muscheln die viel grössere Giftigkeit auf Seiten der Leberextracte, die trotz einer um das 8-10fache kleineren Dosis und trotz grösserer zu den Versuchen verwandter Thiere noch immer viel sicherer und intensiver wirkten, als die zugehörigen Extracte der Weichtheile. Jedenfalls steht diese jetzt mehrmals beobachtete mindere Giftigkeit der Weichtheile in keinem Widerspruch mit der von mir früher festgestellten Localisation des Giftes in der Leber zu der Zeit, als sich die Muscheln auf der Höhe ihrer Giftigkeit befanden.

Auf Grund der vorstehenden Beobachtungen und der vielfach nachgewiesenen Analogien zwischen Muscheln und Seesternen liegt aber auch die Annahme sehr nahe, dass sich auch die Seesterne in den Monaten Januar und Februar dieses Jahres, in denen wir sie zum ersten Mal untersuchten, nicht auf der vollen Höhe ihrer Giftigkeit befanden, sondern dass letztere ebenso, wie die Muscheln im November and December vorigen Jahres, noch viel giftiger waren, als jetzt.

IX. Was nun die ätiologische Seite der Giftfrage anbetrifft, so ist es gewiss von allgemeinstem Interesse, den $\mathrm{Ur}$ sachen nachzuforschen, durch die ein so furchtbares Gift, wie das Muschelgift und das identische Seesterngift, entstehen. Die Thatsachen weisen unzweifelhaft auf eine locale Aetiologie hin. Dies ergiebt sich einmal aus der von verschiedenen Seiten, auch von mir wiederholt constatirten Thatsache, dass die Muscheln anderer Herkunft, z. B. von Norderney, gar nicht giftig sind, sowie aus der ferneren Thatsache, dass sogar in Wilhelmshaven selbst die giftigen Muscheln und Seesterne nar auf ein bestimmtes Gebiet beschränkt sind. Ich habe bereits in meiner früheren Mittheilung l. c. bei der Erörterung der Hypothese von dem "umgebenden Medium“ als Ursache des Giftigwerdens auf die 
Nothwendigkeit hingewiesen, die localen Verhältnisse weiter zu erforschen. In dieser Beziehung ist zuerst der so nahe liegenden und auch verwertheten Annahme entgegenzutreten, dass möglicher Weise die Abflüsse aus der Stadt Wilhelmshaven direct oder indirect das giftige Agens für die Seesterne und Miesmuscheln hergeben. Das ist nicht der Fall. Ich habe bereits Eingangs darauf hingewiesen, dass die Stadt Wilhelmshaven kanalisirt ist und keinerlei Spüljauche aus der Stadt oder Umgebung in den Hafen in seiner ganzen Ausdehnung hineingelangt. In gleicher Weise ist eine Verunreinigung des Binnenhafens durch Fäcalien oder Schiffsabfälle streng verboten. Selbst bei nicht vollkommener Beobachtung dieser Vorschriften wird eine irgendwie erhebliche Ansammlung derartiger Abfälle nicht stattfinden, und man wird daher auf diese Punkte nicht recurriren dürfen als maassgebende Ursache für das Giftigwerden der Muscheln und Seesterne. Es bleibt nur eine locale Ursache übrig, die allerdings in erheblichster Weise in der Wilhelmshavener Anlage Platz greift; das ist die Stagnation des Wassers und, ganz allgemein ausgedrïckt, die daraus nothwendig resultirende Anhäufung schädlicher Stoffe in diesem stagnirenden Wasser. Es hat sich nun bei unsern Versuchen in frappanter Weise eine vollkommene Congruenz zwischen dem Stagnationsgrade des Wassers und der Giftigkeit der Seesterne und Muscheln herausgestellt. Diese Stagnation des Wassers ist im Hafenbassin am grössten, nahezu eine vollkommene; dieselbe nimmt etwas ab, je mehr man sich dem Vorhafen nähert, wo der Wasserwechsel beim Oeffnen und Schliessen der Schlensen etwas grösser ist. Genau entsprechend diesem Grade der Stagnation waren nun aber im Hafenbassin und seinen Anhängen die Seesterne und Miessmuscheln am giftigsten und nahmen nach dem Vorhafen hin an Giftigkeit ab. Auf die wesentliche Bedeutung des stagnirenden Wassers weisen auch die Versuche von Herrn Geheimrath Virchow und Herrn Kreisphysikus Schmidtmann hin. Nicht giftige, von letzterem im Hafenbassin ausgesetzte Muscheln sind in 2-3 Wochen giftig geworden, während umgekehrt giftige Muscheln, in dem freien Seewasser der Hafeneinfahrt ausgesetzt, in derselben Zeit ihre giftigen Eigenschaften vollkommen verloren haben. Dieselbe 
Entgiltung der Muscheln erreichte Herr Geheimrath Virchow auch hier in dem frischen Wasser des Aquarium.

Die weitere Frage war nun aber wieder die, ob in diesem stagnirenden Wasser das Gift, dessen chemische Darstellung aus den Muscheln Brieger gelungen ist, bereits als fertig gebildet anzunehmen ist oder nicht. Ich hatte in meiner früheren Arbeit gegenüber der Hypothese von einem derartigen präformirten Gifte in dem umgebenden Medium und der blossen Aufnahme desselben durch die Muscheln bemerkt, dass die Hauptsache für diese Hypothese noch fehlt, nehmlich der Nachweis eines solchen Giftes in dem umgebenden Medium, das ganz so wirkt, wie die Muscheln bez. die Seesterne selbst. Auch meine jetzigen Versuche, die ich mit Rücksicht auf diese Hypothese Anfangs Januar mit dem stagnirenden Wasser direct angestellt habe, sprechen gegen diese Amahme. Diese Versuche haben ergeben, dass selbst von den Stellen, wo die Muscheln und Seesterne am giftigsten sind, das unter den nothwendigen Cautelen in sterilisirten Gefässen gesammelte Wasser wirkungslos ist. So wurde das Wasser aus dem Hafenbassin je 2 ganz jungen Meerschweinchen in der grossen Dosis von je $7 \mathrm{ccm}$ subcutan injicirt; als das ganz erfolglos war, wurden $70 \mathrm{ccm}$ desselben Wassers auf $5 \mathrm{ccm}$ eingedampft, und dieser concentrirte Rückstand denselben jungen Meersohweinchen zu gleichen Theilen am folgenden Tage ganz beigebracht. Auch hiernach traten keinerlei charakteristische Intoxicationserscheinungen auf; die Thiere bekamen nur in Folge des Salzgehaltes des Wassers locale Reizerscheinungen. Dasselbe Resultat ergab auch das Wasser von einer andern Stelle, aus Dock II; das Wasser war hier trübe und sehr stark schwefelwasserstoffhaltig, wie denn überhaupt ans der Sohle der Docks ein fortwährender Zufluss von Schwefelwasser stattfindet. Auch mit grossen Dosen von diesem Schwefelwasser, von dem zunächst ein grosses Meerschweinchen $10 \mathrm{ccm}$, sodann 2 ganz junge Meerschweinchen je $8 \mathrm{ccm}$ in Pausen subcutan bekommen haben, habe ich keinerlei toxische Wirkung gesehen, auch dann nicht, als ich am folgenden Tage das durch Eindampfen $6 \mathrm{mal}$ concentrirter gemachte Schwefelwasser zu je $3 \mathrm{ccm}$ denselben Thieren beibrachte. In gleicher Weise war dieses Schwefelwasser auch vom Magen aus un- 
wirksam; ein Kaninchen, dem $60 \mathrm{~cm}$ in den Magen eingespritzt waren, blieb vollkommen gesund. Auch das Wasser des Vorhafens brachte keinerlei Erscheinung von Vergiftung hervor.

Die vorstehenden Versuche, die vielleicht noch zu anderen Zeiten mit dem Wasser zu wiederholen sind, lassen die Hypothese von der Präformation des Muschelgiftes in dem umgebenden Medium, dem stagnirenden Wasser, jedenfalls als nicht wahrscheinlich erscheinen. Die negativen Ergebnisse der Versuche bestätigen vielmehr die Anschauung, dass das Gift in den Muscheln und in den Seesternen selbst entsteht. Die weiteren Untersuchungen müssen nun darauf gerichtet sein, zu erforschen, welche Stoffe (Bakterien) des stagnirenden Wassers speciell bei dieser Giftproduction innerhalb des Thierkörpers betheiligt sind.

Auch ein anderer Punkt bedarf noch weiterer Untersuchungen. Es ist immerhin möglich, dass nur eines obiger Seethiere das Gift producirt, das andere aber nur indirect giftig wirkt. In dieser Beziehung hat man, ohne positive Grundlage allerdings, neben allen möglichen anderen kleineren Seethieren auch die Aufnahme von Seesternen als Ursache des Giftigwerdens der Muscheln hier und da bezeichnet. Die letztere Annahme ist aber um so weniger wahrscheinlich, als die Muscheln sich nicht von Seesternen ernähren. Der umgekehrte Fall ist eher möglich, da die Seesterne auch von Weichthieren leben; bemerken möchte ich aber doch, dass ich trotz mehrfacher Untersuchungen niemals ganze Individuen oder Muscheltheile innerhalb der giftigen Seesterne aufgefunden habe.

Was die Fische anbetrifft, so habe ich mit den 4 verschiedenen Fischarten obigen Versuchen zufolge keine Giftwirkungen bekommen; doch sind die Versuche mit den Fischen in Wilhelmshaven noch zu anderen Zeiten zu wiederholen, zumal da zeitweise Fische mit Krankheitserscheinungen in dem Wilhelmshavener Binnenwasser vorkommen. Dasselbe gilt auch von den Garneelen. Ich verweise hier auf das oben mitgetheilte Experiment mit dem alkoholischen Auszug der Garneelen, mit dem wir bei einem Meerschweinchen Intoxicationserscheinungen hervorhrachten, die zwar schnell vorübergingen, aber doch an Miessmuschelvergiftungen erinnerten. Ohne auf dieses eine Experiment hin besondere Schlüsse machen $z u$ wollen, wurde ich 
doch durch dasselbe an die Beobachtungen erinnert, nach denen Garneelen in Deutschland, Holland und Frankreich zu Massenvergiftungen Veranlassung gegeben haben, die in ihren Symptomen ebenso verliefen, wie die menschlichen Vergiftungen durch Miessmuscheln.

Zum Schluss erwähne ich hier noch einge therapeutische Versuche. Bei dem unzweifelhaft paralytischen Charakter, den die Muschel- ebenso wie die Seesternvergiftung, bei unseren Thieren zeigte, war der Versuch gewiss gerechtfertigt, ob man die Thiere nicht durch ein Antiparalyticum, durch Strychnin, am Leben zu erhalten im Stande wäre. Sobald die ersten Intoxicationssymptome eintraten, injicirte ich deshalb $\frac{1}{2}-1 \mathrm{mg}$ Strychninum nitricum subcutan: ich habe aber hiernach keinerlei Erfolge, weder in Bezug auf Schwere der Erscheinungen, noch hinsichtlich des zeitlichen Ablaufes derselben gesehen; alle Thiere gingen ebenso, wie die nicht injicirten, zu Grunde.

Die Miessmuschel- und Seesternfrage ist noch nicht erledigt: manche dunkle Punkte bedürfen noch der Aufklärung. Bei dem aus verschiedenen Gründen berechtigten Interesse der Hafenverwaltung, das Wasser innerhalb der Hafenanlage möglichst constant zu erhalten, ist die Wahrscheinlichkeit vorhanden, dass im Laufe der Sommermonate die Miessmuscheln und Seesterne ihre alte Giftigkeit wieder erlangen. Erneute Untersuchungen werden alsdann vielleicht die noch dunklen Punkte zur Entscheidung bringen.

Herrn Apotheker König in Wilhelmshaven, der jeder Zeit bereit war, mich mit dem zu den vorstehenden Untersuchungen nöthigen Material zu versorgen, sage ich hier meinen besten Dank. 